\title{
Fast Sparse Multinomial Regression Applied to Hyperspectral Data
}

\author{
Janete S. Borges ${ }^{1}$, José M. Bioucas-Dias ${ }^{2}$, and \\ André R.S. Marçal ${ }^{1}$ \\ ${ }^{1}$ Faculdade de Ciências, Universidade do Porto \\ DMA, Rua do Campo Alegre, 687, 4169-007 Porto, Portugal \\ $\{$ jsborges, andre.marcal\}@fc.up.pt \\ ${ }^{2}$ Instituto de Telecomunicações - Instituto Superior Técnico \\ Lisboa, Portugal \\ bioucas@lx.it.pt
}

\begin{abstract}
Methods for learning sparse classification are among the state-of-the-art in supervised learning. Sparsity, essential to achieve good generalization capabilities, can be enforced by using heavy tailed priors/ regularizers on the weights of the linear combination of functions. These priors/regularizers favour a few large weights and many to exactly zero. The Sparse Multinomial Logistic Regression algorithm [1] is one of such methods, that adopts a Laplacian prior to enforce sparseness. Its applicability to large datasets is still a delicate task from the computational point of view, sometimes even impossible to perform. This work implements an iterative procedure to calculate the weights of the decision function that is $O\left(\mathrm{~m}^{2}\right)$ faster than the original method introduced in [1] ( $m$ is the number of classes). The benchmark dataset Indian Pines is used to test this modification. Results over subsets of this dataset are presented and compared with others computed with support vector machines.
\end{abstract}

\section{Introduction}

In the recent years the increase in spectral resolution of airborne and spaceborne sensors has led to the availability of hyperspectral images, with hundreds of contiguous narrow spectral bands. This type of images provide detailed information about spectral signatures, which should make possible discrimination among a larger set of classes [2]. Most image processing algorithms were developed for multi-spectral images, amongst of them the supervised classification algorithms. The increase of data dimensionality, from multispectral to hyperspectral images, brought problems related with the Hughes phenomenon that common algorithms can not manage. The impossibility, or high cost, of having sufficient training samples to keep an acceptable classification error, fostered the development of classification methods capable to deal with high volume/high dimensional datasets.

Support Vector Machines (SVMs) are state-of-the-art in discriminative supervised classification. The SVM approach aims at expressing a classification

A. Campilho and M. Kamel (Eds.): ICIAR 2006, LNCS 4142, pp. 700709 2006.

(C) Springer-Verlag Berlin Heidelberg 2006 
task by explicitly optimizing a measure of the generalization capability of the learning machine 3 . SVMs have been successfully used for hyperspectral data classification due to their ability to deal with large input spaces efficiently and produce sparse solutions. One example of such an application is the work developed by Camps-Valls et. al 4 . They present a comparative study of kernel-based methods in terms of robustness to noise, input space dimension, and number of labelled samples. Their conclusion is that, when compared with other kernel methods, SVMs shows excellent results in terms of computational cost, accuracy, robustness to noise, and sparsity.

Recently, sparseness has been exploited as a way to control the generalization capability of a learning machine (see, e.g., [5, 6], 7], 8], 9], 10]). In these methods, the classifier depends on a sparse linear combination of basis functions, which may be any function (linear or not) of the features, including kernels centered on the training samples.

In a large array of experiments, sparse classifiers have shown state-of-the-art performance in supervised learning. The Sparse Multinomial Logist Regression (SMLR) algorithm [1] is one of such sparse methods that applies to multiclass and adopts a Lapacian prior to enforce sparseness. For a sample of size $n, d$-dimensional features, and $m$ classes, the computational cost of SMLR is $O\left((d m)^{3}\right)$ or $O(n d m)$, depending on the implementation. In practice, we have verified that only the former, corresponding to a reweighed least square type algorithm, is applicable with kernels and large number of samples.

In the case of kernels, we have $d=n$ and the computational cost of the SMRL algorithm is $O\left((\mathrm{~nm})^{3}\right)$, which is basically the cost of solving a system of size $\mathrm{nm}$. This complexity is unbearable in many problems of moderate number of samples and many classes. For example, if we have a matrix of size $n=1000$ samples and ten classes, $m=10$, there is the need of solve a system of 10000 equations in each iteration of the SMRL algorithm.

A solution for this problem is a modification to the iterative method used in Sparse Multinomial Logistic Regression (SMLR), which results in a faster and more efficient algorithm. The modification consists in using the Block GaussSeidel method [11] to solve a linear system. In each iteration, instead of solving the complete set of weights, only blocks corresponding to the weights belonging to the same class are solved. Details of this matter are available in [12. In practice, in each iteration we solve $m$ systems of dimension equal to the number of samples, thus, resulting in an improvement of the order of $O\left(\mathrm{~m}^{2}\right)$.

\section{Sparse Algorithms}

The sparse method used here is, basically, that of Krishnapuram et. al [1, but uses the Block Gauss-Seidel iterative scheme to solve the linear system implied by the quadratic majorizer of the Lapacian prior. In this section the method proposed by Krishnapuram et. al - Sparse Multinomial Logistic Regression [1] is briefly reviewed. Then, the Block Gauss-Seidel iterative scheme is described. 


\subsection{Sparse Multinomial Logistic Regression}

The SMLR algorithm learns a multi-class classifier based on the multinomial logistic regression. By incorporating a Laplacian prior, this method performs simultaneously feature selection, to identify a small subset of the most relevant features, and learns the classifier itself.

Let $x=\left[x_{1}, \ldots, x_{d}\right]^{T} \in R^{d}$ be the $d$ observed features. The goal is to assign to each $x$ the probability of belonging to each of the $m$ classes, given $m$ sets of feature weights, one for each class. In particular, if $y=\left[y^{(1)}, \ldots, y^{(m)}\right]^{T}$ is a 1-of$\mathrm{m}$ encoding of the $m$ classes, and if $w^{(i)}$ is the feature weight vector associated with class $i$, then the probability that a given sample $x$ belongs to class $i$ is given by

$$
P\left(y^{(i)}=1 \mid x, w\right)=\frac{\exp \left(w^{(i)^{T}} x\right)}{\sum_{i=1}^{m} \exp \left(w^{(i)^{T}} x\right)}
$$

where $w=\left[w^{(i)^{T}}, \ldots, w^{(m)^{T}}\right]^{T}$. Usually a Maximum Likelihood (ML) estimation procedure is used to obtain the components of $w$ from the training data, simply by maximizing the log-likelihood function [13]:

$$
l(w)=\sum_{j=1}^{n}\left[\sum_{i=1}^{m} y_{j}^{(i)} w^{(i)^{T}} x_{j}-\log \sum_{i=1}^{m} \exp \left(w^{(i)^{T}} x_{j}\right)\right] .
$$

A sparsity promoting $l_{1}$ prior on the entries of $w$ is incorporated, in order to achieve sparsity in the estimate of $w$. With this modification, a Maximum A Posteriori (MAP) is used instead of typical ML criterion for multinomial logistic regression. The estimates of $w$ are then given by:

$$
\hat{w}_{M A P}=\arg \max _{w} L(w)=\arg \max _{w}[l(w)+\log p(w)]
$$

where $p(w)$ is the Laplacian prior on $w$, which means that $p(w) \propto \exp \left(-\lambda\|w\|_{1}\right)$ where $\lambda$ acts as a tunable regularization parameter.

The inclusion of a Laplacian prior does not allow the use of the classical IRLS method. The bound optimization framework supplies us with a tool to tackle this optimization problem. The central concept in bound optimization is the iterative replacement of the funtion to be optimized, in our case $L(w)=l(w)+\log p(w)$, with a surrogate function $Q$ [14], such that,

$$
L\left(w^{(t+1)}\right) \geq L\left(w^{(t)}\right)
$$

and

$$
w^{(t+1)}=\arg \max _{w} Q\left(w \mid \widehat{w}^{(t)}\right) .
$$

Since $l(w)$ is concave and $C^{2}$, a surrogate function for it, $Q_{l}\left(w \mid \widehat{w}^{\prime}\right)$, can be determined by using a bound on its Hessian $H$. Let $B$ be a negative definite matrix such that $H(w)-B$ is positive semi-definite, i.e., $H(w) \succ B$ for any $w$. A valid surrogate function is

$$
Q\left(w \mid \hat{w}^{(t)}\right)=w^{T}\left(g\left(\hat{w}^{(t)}\right)-B \hat{w}^{(t)}\right)+\frac{1}{2} w^{T} B w
$$


where

$$
B \equiv-\frac{1}{2}\left[I-11^{T} / m\right] \otimes \sum_{j=1}^{n} x_{j} x_{j}^{T}
$$

and $1=[1,1, \ldots, 1]^{T}, g(w)$ is the gradient of $l(w)$ given by

$$
g(w)=\sum_{j=1}^{n}\left(y_{j}^{\prime}-p_{j}(w)\right) \otimes x_{j},
$$

with $y_{j}^{\prime}=\left[y_{j}^{(1)}, y_{j}^{(2)}, \ldots, y_{j}^{(m-1)}\right]^{T}$ and $p_{j}(w)=\left[p_{j}^{(1)}(w), \ldots, p_{j}^{(m-1)}(w)\right]^{T}$.

Concerning the $l_{1}$ norm $\|w\|_{1}=\sum_{i}\left|w_{i}\right|$, we note that, for a given $w_{i}^{(t)},-\left|w_{i}\right| \geq$ $-1 / 2 w_{i}^{2} /\left|w_{i}^{(t)}\right|+c^{t e}$, where $c^{t e}$ is a constant. Thus, both terms of $L(w)$ have a quadratic bound. Since the sum of functions is lower bounded by the sum of the correspondent lower bounds, we have a quadratic bound for $L(w)$, given by

$$
Q\left(w \mid \widehat{w}^{(t)}\right)=w^{T}\left(g\left(\hat{w}^{(t)}\right)-B \hat{w}^{(t)}\right)+\frac{1}{2} w^{T} B w+\frac{1}{2} w^{T} \Lambda^{(t)} w
$$

where

$$
\Lambda^{(t)}=\operatorname{diag}\left\{\left|\hat{w}_{1}^{(t)}\right|^{-1}, \ldots,\left|\hat{w}_{d(m-1)}^{(t)}\right|^{-1}\right\} .
$$

The maximization of (10) leads to

$$
\hat{w}^{(t+1)}=\left(B-\lambda \Lambda^{(t)}\right)^{-1}\left(B \hat{w}^{(t)}-g\left(\hat{w}^{(t)}\right)\right) .
$$

Numerically, Eq11] is equivalent to solve [1]:

$$
\hat{w}^{(t+1)}=\Gamma^{(t)}\left(\Gamma^{(t)} B \Gamma^{(t)}-\lambda I\right)^{-1} \Gamma^{(t)}\left(B \hat{w}^{(t)}-g\left(\hat{w}^{(t)}\right)\right),
$$

where

$$
\Gamma^{(t)}=\operatorname{diag}\left\{\left|\hat{w}_{1}^{(t)}\right|^{1 / 2}, \ldots,\left|\hat{w}_{d(m-1)}^{(t)}\right|^{1 / 2}\right\}
$$

It is now possible to perform exact MAP multinomial logistic regression under a Laplacian prior, with the same cost as the original IRLS algorithm for ML estimation (see [11]).

\subsection{Fast Sparse Multinomial Logistic Regression}

The computational cost of $O\left((d m)^{3}\right)$ to solve the linear system in (12) at each iteration is often prohibitive. This happens when large datasets are processed, either because the original number of features is very large, or because a very large training dataset is used.

A solution for this problem is a modification to the iterative method used in SMLR, which results in a faster and more efficient algorithm. The modification 
consists in using the Block Gauss-Seidel method [11] to solve the system used in the IRLS method. In each iteration, instead of solving the complete set of weights, only blocks corresponding to the weights belonging to the same class are solved. Details of this matter are available in 12 . In practice, in each iteration we solve $m$ systems of dimension equal to the number of samples, thus, resulting in an improvement of the order of $O\left(\mathrm{~m}^{2}\right)$.

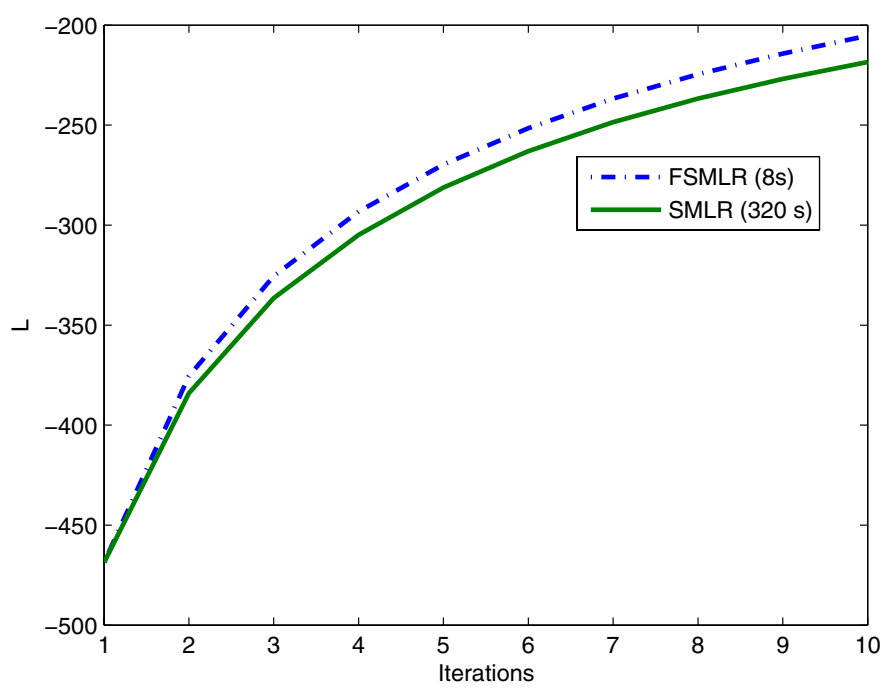

Fig. 1. Evolution of energy (??) when computed with (12) and with the Block GaussSeidel iterative scheme proposed in [12. Notice the huge difference in time for a problem with $n=500, d=500$, and $m=9$. FSMRL takes 8 seconds, whereas SMRL takes 320 seconds.

Figure1 1illustrates the gain in computational cost of the proposed fast SMLR (FSMRL) for a problem with $n=500, d=500$, and $m=9$ on a $2 \mathrm{GHz}$ PC. Only the first ten iterations are shown. Notice that for a very similar energy, SMRL takes 320 seconds, whereas FSMRL takes 8 seconds.

\section{$3 \quad$ Experimental Results}

\subsection{Data Description}

Experiments are performed with an AVIRIS spectrometer image, the Indian Pines 92 from Northern Indiana, taken on June 12, 1992 [15. The ground truth data image consists of $145 \times 145$ pixels of the AVIRIS image in 220 contiguous spectral bands, at $10 \mathrm{~nm}$ intervals in the spectral region from 0.40 to $2.45 \mu \mathrm{m}$, at a spatial resolution of $20 \mathrm{~m}$. Four of the 224 original AVIRIS bands contained 
no data or zero values and were thus removed. The image covers an agricultural portion of North-West Indiana with 16 identified classes. Due to the insufficient number of training samples, seven classes were discarded, leaving a dataset with 9 classes distributed by 9345 elements. This dataset was randomly divided into a set of 4757 training samples and 4588 validation samples. The number of samples per class and the class labels are presented in table 3.1 and their spatial distribution in figure 2 .
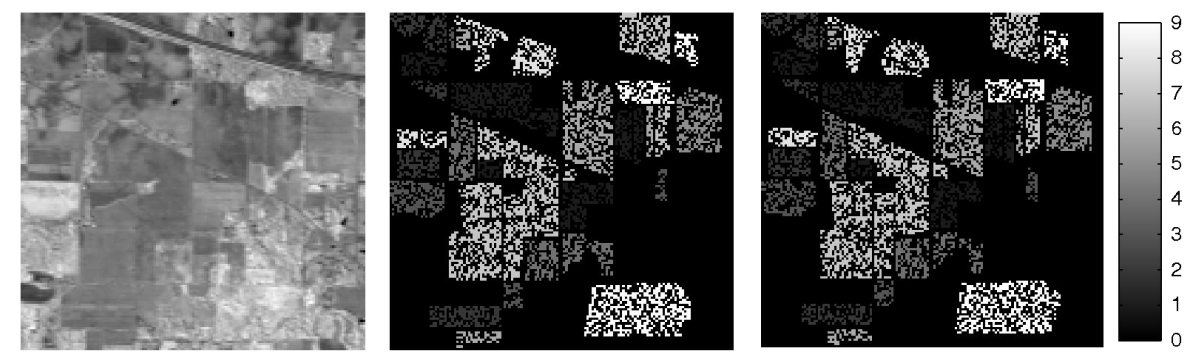

Fig. 2. AVIRIS image used for testing. Left: original image band 50 (near infrared); Centre: training areas; Right: validation areas.

Table 1. Number of training and validation samples used in the experiments

\begin{tabular}{lcc}
\hline Class & Training & VALIDATION \\
\hline C1 - Corn-no till & 742 & 692 \\
C2 - Corn-min till & 442 & 392 \\
C3 - Grass/Pasture & 260 & 237 \\
C4 - Grass/Trees & 389 & 358 \\
C5 - Hay-windrowed & 236 & 253 \\
C6 - Soybean-no till & 487 & 481 \\
C7 - Soybean-min till & 1245 & 1223 \\
C8 - Soybean-clean till & 305 & 309 \\
C9 - Woods & 651 & 643 \\
\hline
\end{tabular}

\subsection{Experimental Setup}

Two different types of classifiers using linear and RBF kernels were evaluated in different conditions: (i) using $10 \%, 20 \%$ and $100 \%$ of the training samples to learn a linear kernel, (ii) using 10\%, 20\% and 50\% of the training samples to learn a RBF kernel. The six classifiers were applied to (i) the entire set of bands, and (ii) discarding 20 noisy bands (104-108, 150-163, 220), resulting on 12 scenarios. These noisy bands correspond to the spectral regions where there is significant absorption of radiation by the atmosphere due to water vapour. The two types of classifiers have parameters that are defined by the user. In the case of linear kernel, only the $\lambda$ parameter has to be tuned. Together with $\lambda$, the RBF kernel has also the parameter $\sigma$ that should be tuned. 
The tuning process was done by first dividing the training set into a subset with approximately $10 \%$ of training samples, which was used to learn the classifier, and the remaining $90 \%$ used to compute an estimate of the Overall Accuracy $(\mathrm{OA})$. This process was repeated 20 times in order to obtain the parameter that maximizes the OA in the remaining training sets. Since the RBF kernel had two parameters to be tuned, we first looked for the best $\sigma$ in one subset and then repeated the same process using the $\sigma$ achieved. Several values for $\lambda$ and $\sigma$ were tested: for the linear kernel $\lambda=16,18,20,22,24$; for the RBF kernel $\lambda=0.0004,0.00045,0.0005,0.00055,0.0006$ and $\sigma=0.48,0.54,0.6,0.66,0.72$.

Table 2. Overall accuracy of a RBF kernel classification, measured on a subset of the training dataset

\begin{tabular}{cccccc}
\hline$\lambda / \sigma$ & 0.48 & 0.54 & 0.6 & 0.66 & 0.72 \\
\hline 0.0004 & $85.06 \%$ & $85.53 \%$ & $85.37 \%$ & $84.93 \%$ & $84.40 \%$ \\
0.00045 & $85.14 \%$ & $85.56 \%$ & $85.32 \%$ & $84.98 \%$ & $84.38 \%$ \\
0.0005 & $85.24 \%$ & $85.50 \%$ & $85.32 \%$ & $84.82 \%$ & $84.27 \%$ \\
0.00055 & $85.45 \%$ & $85.43 \%$ & $85.22 \%$ & $84.66 \%$ & $84.43 \%$ \\
0.0006 & $85.48 \%$ & $85.40 \%$ & $84.95 \%$ & $84.56 \%$ & $84.38 \%$ \\
\hline
\end{tabular}

In table 3.2 an example of the tuning process over one subset of $20 \%$ of the training samples and 220 spectral bands is showed. In this example we take $\sigma=0.00054$ as the best $\sigma$. Then we fixed this value and looked for the best $\lambda$ running 20 times the same procedure for different subsets of the same size. The same process was carried out to achieve the best $\lambda$ and $\sigma$ using $10 \%$ of the training set for RBF kernel.

When dealing with the complete training set and linear kernel, a crossvalidation procedure was performed for $\lambda=16,18,20,22,24$. The implementation of the method presented was done in Matlab 16, which unfortunately has several limitations when dealing with large datasets. Therefore, it was not possible to perform the learning task with a RBF kernel using the complete training set. In the case of RBF kernel, only $50 \%$ of the training samples were used. The best parameters identified by subsets with $20 \%$ of training samples were selected to perform a classification with RBF kernel over the validation dataset.

\subsection{Results}

The results presented in this section are the overall accuracy measure in the independent (validation) dataset with 4588 samples. In tables 3.3 and 3.3 , the parameters used for each experimental scenario and respective number of support vectors are presented. As one can see, there is in fact a large reduction on the number of features needed to built the classifier. In the case of linear kernel, and using the entire training set, we can see a significant reduction of the number of training samples required to build the classifier. In the RBF case, the reduction is not so great but it is still meaningful. 
Table 3. Best $\lambda$ and number of support vectors (SV) used with linear kernel

\begin{tabular}{c|ccc|ccc}
\hline & \multicolumn{3}{|c|}{220 BANDS } & \multicolumn{3}{c}{200 BANDS } \\
& $10 \%$ & $20 \%$ & $100 \%$ & $10 \%$ & $20 \%$ & $100 \%$ \\
\hline$\lambda$ & 16 & 22 & 18 & 16 & 18 & 18 \\
$\mathrm{SV}$ & 28 & 22 & 85 & 24 & 39 & 37 \\
\hline
\end{tabular}

Table 4. Best $\lambda$ and $\sigma$ and number of support vectors (SV) used with RBF kernel

\begin{tabular}{c|cc|cc}
\hline & \multicolumn{2}{|c|}{220 BANDS } & \multicolumn{2}{c}{200 BANDS } \\
& $10 \%$ & $20 \%$ & $10 \%$ & $20 \%$ \\
\hline$\lambda$ & 0.0005 & 0.0006 & 0.0004 & 0.0006 \\
$\sigma$ & 0.72 & 0.54 & 0.48 & 0.6 \\
SV & 410 & 689 & 570 & 748 \\
\hline
\end{tabular}

Knowing that 20 of the 220 original spectral bands are noisy bands, experiments were carried out with and without those bands. The objective was to observe the influence of a coarse feature selection on the classifiers performance. Tables 3.3 and 3.3 present the $\mathrm{OA}(\%)$ for each case. The improvement in OA due to the coarse selection is not significant. In some cases, the use of all 220 bands gives better results than with 200 bands (without the noisy bands). However, it is worth noting that the differences are not significant in both cases.

Table 5. Results with linear kernel using 10\%, $20 \%$ and the complete training set

\begin{tabular}{cccc}
\hline & $10 \%$ & $20 \%$ & $100 \%$ \\
\hline 220 bands & $76.55 \%$ & $79.69 \%$ & $85.77 \%$ \\
200 bands & $75.57 \%$ & $81.60 \%$ & $85.24 \%$ \\
\hline
\end{tabular}

In order to better evaluate our results, a comparison was made with the results obtained with other kernel-based methods in the same dataset [4. Although there were some limiting factors in the pratical application of the proposed method, due to limitations in Matlab processing capacity, the results obtained are very encouraging. The performance of FSMLR linear proved to be superior to Linear Discriminant Analysis (LDA) [4 as it is summarised in table 3.3. Regarding the use of RBF kernels, our results are about the same as those from [4]. The values presented in 3.3 for SVM-RBF are approximate values extracted from graphical data presented in figure 6 of [4]. Although for RBF kernels our method did not outperform the method used in [4, the sparsity of FSMLR can be an advantage for large datasets. 
Table 6. Results with RBF kernel using 10\%, $20 \%$ and $50 \%$ of training samples

\begin{tabular}{lccc}
\hline & $10 \%$ & $20 \%$ & $50 \%$ \\
\hline 220 bands & $82.93 \%$ & $87.12 \%$ & $90.12 \%$ \\
200 bands & $84.98 \%$ & $86.73 \%$ & $90.52 \%$ \\
\hline
\end{tabular}

Table 7. Comparison of the proposed method with the results from 4

\begin{tabular}{lcccc}
\hline & SMLR LINEAR & LDA [4] & SMLR RBF (50\%) & SVM-RBF (50\%) [ [] \\
\hline 220 bands & $85.77 \%$ & $82.32 \%$ & $90.12 \%$ & $\simeq 91 \%$ \\
200 bands & $85.24 \%$ & $82.08 \%$ & $90.52 \%$ & $\simeq 91 \%$ \\
\hline
\end{tabular}

\section{Conclusion}

In this work the Block Gauss-Seidel method for solving systems is introduced in the SMLR classification algorithm. This approach turns the SMLR a faster and more efficient algorithm for the classification of hyperspectral images. The performance of this technique was tested using a benchmarked dataset (Indian Pines) with nine classes and thousands of labelled samples.

Although the experiments performed in this work were suboptimal, the results proved to be quite satisfactory when compared with the ones achieved by CampsValls et. al 4]. Results with linear kernels were better than the ones achieved with LDA method in [4. Approximately the same results for RBF kernels were obtained with our method and by Camps-Valls [4], using only $50 \%$ of the dataset and without tuning all the parameters.

The preliminary results presented in this paper are encouraging as a starting point for the inclusion of statistical spatial information in classification of hyperspectral images. Future work can be developed to include the spatial context, in the sense that neighbouring labels are more likely to have similar labelling. Plans for future work also include the development of semi-supervised techniques based on the FSMLR method proposed.

\section{Acknowledgments}

The first author would like to thank the Fundação para a Ciência e a Tecnologia (FCT) for the financial support (PhD grant SFRH/BD/17191/2004). The authors would also like to thank David Landgrebe for providing the AVIRIS data.

\section{References}

1. Krishnapuram, B. Carin, L. Figueiredo, M.A.T. and Hartemink, A.J.: Sparse Multinomial Logistic Regression: Fast Algorithms and Generalization Bounds. IEEE Transactions on Pattern Analysis and Machine Intelligence, Vol. 27, Issue 6. (2005) 957-968. 
2. Landgrebe, D.A. : Signal Theory Methods in Multispectral Remote Sensing. John Wiley and Sons, Inc., Hoboken, New Jersey. (2003)

3. Vapnik, V. : Statistical Learning Theory. John Wiley, New York. (1998)

4. Camps-Valls, G. and Bruzzone, L. : Kernel-based methods for hyperspectral image classification. IEEE Transactions on Geoscience and Remote Sensing, Vol. 43, Issue 6. (2005) 1351-1362.

5. Tipping, M. : Sparse Bayesian learning and the relevance vector machine. Journal of Machine Learning Research, Vol. 1. (2001) 211-244.

6. Figueiredo, M. : Adaptive Sparseness for Supervised Learning. IEEE Transactions on Pattern Analysis and Machine Intelligence, Vol. 25, no. 9. (2003) 1150-1159.

7. Csato, L. and Opper, M. : Sparse online Gaussian processes. Neural Computation, Vol. 14, no. 3. (2002) 641-668.

8. Lawrence, N.D. Seeger, M. and Herbrich, R.: Fast sparse Gaussian process methods: The informative vector machine. In: Becker, S. Thrun, S. and Obermayer, K. (eds.): Advances in Neural Information Processing Systems 15. MIT Press, Cambridge, M.A. (2003) 609-616.

9. Krishnapuram, B. Carin, L. and Hartemink, A.J.: Joint classifier and feature optimization for cancer diagnosis using gene expression data. Proceedings of th International Conference in Research in Computational Molecular Biology (RECOMB'03) Berlin, Germany (2003).

10. Krishnapuram, B. Carin, L. Hartemink, A.J. and Figueiredo, M.A.T. : A Bayesian approach to joint feature selection and classifier design. IEEE Transactions on Pattern Analysis and Machine Intelligence, Vol. 26. (2004) 1105-1111.

11. A.Quarteroni, R.Sacco and F.Saleri: Numerical Mathematics. Springer-Verlag, New-York. (2000)TAM Series n. 37..

12. Bioucas Dias, J.M. : Fast Sparse Multinomial Logistic Regression - Technical Report. Instituto Superior Técnico. Available at http://www.lx.it.pt/〜bioucas/ (2006).

13. Hastie, T., R. Tibshirani, and J. Friedman: The Elements of Statistical Learning Data Mining, Inference and Prediction. Springer, New York. (2001).

14. Lange, K., Hunter, D. and Yang, I. : Optimizing transfer using surrogate objective functions. Journal of Computational and Graphical Statistics, Vol. 9. (2000) 1-59.

15. Landgrebe, D.A. : NW Indiana's Indian Pine (1992). Available at http://dynamo.ecn.purdue.edu/ ${ }^{\sim}$ biehl/MultiSpec/.

16. The MathWorks : MATLAB The Language of Technical Computing - Using MATLAB : version 6. The Math Works, Inc. (2000) 